\title{
ON SOME PROPERTIES OF THREE PARAMETERS MITTAG-LEFFLER FUNCTIONS
}

\author{
İbrahim Aktaş ${ }^{\mathrm{a}^{*}}$ (D) , Ayşe Topbaş ${ }^{\mathrm{b}}$ \\ $a^{a^{*}}$ Department of Mathematics, Kamil Özdă̆ Science Faculty, University of Karamanoğlu Mehmetbey, \\ Karaman, Turkey \\ aktasibrahim38@gmail.com (*corresponding author) \\ ${ }^{b}$ Abdullah Tayyar Anatolian High School, Beyazkent Neighborhood, Karaman, Turkey \\ aysetopbas34@gmail.com
}

\begin{abstract}
In this paper, our main aim is to discuss some monotonic and logarithmic concavity properties of threeparameter Mittag-Leffler function by using its Weierstrassian product representation and some earlier results on power series. In addition, by using the relationships between Mittag-Leffler type functions and some basic functions, we give some specific examples related to the monotonic and logarithmic concavity properties of some trigonometric and hyperbolic functions.
\end{abstract}

Keywords: Mittag-Leffler function, real zeros of Mittag-Leffler function, monotonicity, logarithmic concavity

\section{Introduction and preliminaries}

Studying on monotonic and geometric properties of certain special functions has become very attractive for mathematicians since their usefulness in solving problems on applied sciences. As a result of this attraction many authors began to investigate monotonic and geometric properties of some special functions such as Bessel, Struve, Lommel, Wright, Hypergeometric functions and their some analogous(see [1] and [2] for examples and references there in). In the literature, undoubtedly that one of the most important special function is the Mittag-Leffler function. This function is a generalization of the famous exponential function as well as it arises in solving on biological, physical, engineering and earth sciences problems. Due to its vast potential in the applied sciences the Mittag-Leffler function and its generalizations is still studying intensively by the many mathematicians. It is known that the exponential function arises in the solution of integer order differential equations, while the Mittag-Leffler function 
has a similar role in the solution of non-integer order differential equations. The historical overview and detailed information about the Mittag-Leffler function may be found in [3, 4].

The outcomes of this paper is as follow: In the rest of Section 1 we give the definitions of Mittag-Leffler functions and remind some interesting properties like reality of the zeros and infinite product representation of three-parameter Mittag Leffler function. Also, we remind a useful lemma to prove monotonic properties and give the definition of logarithmic concavity of a function. In Section 2, we give our main results and their consequences, while the Section 3 is devoted for some applications of our main results.

Now, we would like to remind the definition of Mittag-Leffler functions and their some properties. In 1903, Gösta Magnus Mittag-Leffler defined the one-parameter Mittag-Leffler function by the following power series:

$$
\phi(\alpha, x)=\sum_{n \geq 0} \frac{x^{n}}{\Gamma(\alpha n+1)}, \quad \alpha \in \mathbb{C}, \quad \Re(\alpha)>0,
$$

where $\Gamma$ denotes the Euler's gamma function. It can be easily seen that this function is a generalization of the exponential function. In order to see this it is enough to write $(\alpha n) !=$ $\Gamma(\alpha n+1)$ in the denominator. Later on, Wiman [9] introduced another function called twoparameter Mittag-Leffler function with the similar properties as follow:

$$
\phi(\alpha, \beta, x)=\sum_{n \geq 0} \frac{x^{n}}{\Gamma(\alpha n+\beta)}, \quad \alpha, \beta \in \mathbb{C}, \quad \Re(\alpha)>0 .
$$

In 1971, three-parameter Mittag-Leffler function (or so-called Prabhakar's function) was introduced by Prabhakar [8] in the following form:

$$
\phi(\alpha, \beta, \gamma, x)=\sum_{n \geq 0} \frac{(\gamma)_{n} x^{n}}{n ! \Gamma(\alpha n+\beta)}, \quad \alpha, \beta, \gamma \in \mathbb{C}, \quad \Re(\alpha)>0, \quad \Re(\beta)>0
$$

where $(\gamma)_{n}$ denotes the Pochhammer symbol and it is defined by $(a)_{n}=\frac{\Gamma(a+n)}{\Gamma(a)}$. It is important to remind here that by giving certain special values to the parameters $\alpha, \beta$ and $\gamma$ in the function $x \mapsto \phi(\alpha, \beta, \gamma, x)$, it is possible to obtain some trigonometric and hyperbolic functions.

In the present paper, our main aim is to investigate the sign of the three-parameter MittagLeffler function. Also, we will show that the mentioned function is a decreasing function on an interval determined by its first positive zero. Further, we will discuss logarithmic concavity of this function in the certain sets defined by its real zeros. Finally, we will define a new function by using three-parameter Mittag-Leffler function and its derivative, and investigate its monotonicity on $\mathbb{R}^{+}$. For this purpose, we remember some interesting properties of threeparameter Mittag-Leffler function read as follow.

In order to state their some results, Baricz and Prajapati [1] defined the following three transformations mapping the set

$$
\left\{\left(\frac{1}{\alpha}, \beta\right): \alpha>1, \beta>0\right\}
$$


into itself:

$$
\begin{gathered}
A:\left(\frac{1}{\alpha}, \beta\right) \rightarrow\left(\frac{1}{2 \alpha}, \beta\right), B:\left(\frac{1}{\alpha}, \beta\right) \rightarrow\left(\frac{1}{2 \alpha}, \alpha+\beta\right), \\
C:\left(\frac{1}{\alpha}, \beta\right) \rightarrow\left\{\begin{array}{l}
\left(\frac{1}{\alpha}, \beta-1\right), \text { if } \beta>1 \\
\left(\frac{1}{\alpha}, \beta\right), \text { if } 0<\beta \leq 1
\end{array}\right.
\end{gathered}
$$

Putting $W_{b}=A\left(W_{a}\right) \cup B\left(W_{a}\right)$, where $W_{a}=\left\{\left(\frac{1}{\alpha}, \beta\right): 1<\alpha<2, \beta \in[\alpha-1,1] \cup[\alpha, 2]\right\}$, the authors denoted by $W_{i}$ the smallest set containing $W_{b}$ and invariant with respect to transformations mapping $A, B$ and $C$. It is important to emphasize here that with the help of a result of Peresyolkova [7], Kumar and Pathan [5] showed that if $\left(\frac{1}{\alpha}, \beta\right) \in W_{i}$ and $\gamma>0$, then all the zeros of the three-parameter Mittag-Leffler function $\phi(\alpha, \beta, \gamma, x)$ are real and simple. The next Lemma 1.1 which will be used in the proof include an infinite product representation and interlacing properties of the real zeros for the three-parameter Mittag-Leffler function $\phi\left(\alpha, \beta, \gamma,-x^{2}\right)$ and it is proven by Baricz and Prajapati in [1].

Lemma 1.1 (see [1]) If $\left(\frac{1}{\alpha}, \beta\right) \in W_{i}$ and $\gamma>0$, then the function $x \mapsto \phi\left(\alpha, \beta, \gamma,-x^{2}\right)$ has infinitely many zeros which are all real. Denoting by $\lambda_{\alpha, \beta, \gamma, n}$ the $n$th positive zero of $x \mapsto$ $\phi\left(\alpha, \beta, \gamma,-x^{2}\right)$ under the same conditions the Weierstrassian decomposition

$$
\phi\left(\alpha, \beta, \gamma,-x^{2}\right)=\frac{1}{\Gamma(\beta)} \prod_{n \geq 1}\left(1-\frac{x^{2}}{\lambda_{\alpha, \beta, \gamma, n}^{2}}\right)
$$

is valid. Moreover, if $\xi_{\alpha, \beta, \gamma, n}$ denotes the $n$th positive zero of $\Psi^{\prime}(\alpha, \beta, \gamma, x)$, where $\Psi(\alpha, \beta, \gamma, x)=x^{\beta} \phi\left(\alpha, \beta, \gamma,-x^{2}\right)$, then the positive zeros $\lambda_{\alpha, \beta, \gamma, n}$ and $\xi_{\alpha, \beta, \gamma, n}$ are interlaced.

In addition, the following Lemma 1.2 given by Biernacki and Krzyż (see [2]) will be used to prove some monotonic properties of the mentioned functions.

Lemma 1.2 (see [2]) Consider the power series $f(x)=\sum_{n \geq 0} a_{n} x^{n}$ and $g(x)=\sum_{n \geq 0} b_{n} x^{n}$, where $a_{n} \in \mathbb{R}$ and $b_{n}>0$ for all $n \in\{0,1, \ldots\}$, and suppose that both converge on $(-r, r), r>$ 0 . If the sequence $\left\{\frac{a_{n}}{b_{n}}\right\}_{n \geq 0}$ is increasing(decreasing), then the function $x \mapsto\left(\frac{f(x)}{g(x)}\right)$ is also increasing(decreasing) on $(0, r)$.

Here, it is important to note that the above result remains true for the even or odd functions. Finally, the definition of logarithmic concavity of a function can be given as follow:

Definition 1.3 (see[6]) A function $f$ is said to be log-concave on interval $(a, b)$ if the function $\log f$ is a concave function on $(a, b)$.

In order to prove the logarithmic concavity of a function $f$ on the interval $(a, b)$, it is sufficient to show that one of the following two conditions hold true:

1. $\frac{f^{\prime}}{f}$ monotone decreasing on $(a, b)$.

2. $(\log f)^{\prime \prime}<0$. 


\section{Main results}

In this section, we present our main results and their consequences.

Theorem 2.1 Let $\left(\frac{1}{\alpha}, \beta\right) \in W_{i}, \gamma>0$ and denote the $n$th positive zero of the three-parameter Mittag-Leffler function $\phi\left(\alpha, \beta, \gamma,-x^{2}\right)$ by $\lambda_{\alpha, \beta, \gamma, n}$. Further, consider the following sets:

$$
A_{1}=\bigcup_{n \geq 1}\left(\lambda_{\alpha, \beta, \gamma, 2 n-1}, \lambda_{\alpha, \beta, \gamma, 2 n}\right), \quad A_{2}=\bigcup_{n \geq 1}\left(\lambda_{\alpha, \beta, \gamma, 2 n}, \lambda_{\alpha, \beta, \gamma, 2 n+1}\right)
$$

and

$$
A_{3}=\left[0, \lambda_{\alpha, \beta, \gamma, 1}\right) \cup A_{2}
$$

Then, the three-parameter Mittag-Leffler function $\Phi(\alpha, \beta, \gamma, x)=\Gamma(\beta) \phi\left(\alpha, \beta, \gamma,-x^{2}\right)$ has the following properties:

i. $\quad$ the function $x \mapsto \Phi(\alpha, \beta, \gamma, x)$ is negative on $A_{1}$ and it is positive on $A_{3}$,

ii. the function $x \mapsto \Phi(\alpha, \beta, \gamma, x)$ is a decreasing function on $\left(0, \lambda_{\alpha, \beta, \gamma, 1}\right)$,

iii. the function $x \mapsto \Phi(\alpha, \beta, \gamma, x)$ is strictly logarithmic concave on $A_{3}$.

Proof. $i$. In order to prove our assertion, firstly, we consider the Weierstrassian product representation of the three-parameter Mittag-Leffler function $\phi\left(\alpha, \beta, \gamma,-x^{2}\right)$ given by (4). Thus, the function $x \mapsto \Phi(\alpha, \beta, \gamma, x)$ can be written as the following infinite product:

$$
\Phi(\alpha, \beta, \gamma, x)=\prod_{n \geq 1}\left(1-\frac{x^{2}}{\lambda_{\alpha, \beta, \gamma, n}^{2}}\right)=\mathcal{P}_{n} \mathcal{Q}_{n}
$$

where

$$
\mathcal{P}_{n}=\prod_{n \geq 1}\left(\frac{\lambda_{\alpha, \beta, \gamma, n}+x}{\lambda_{\alpha, \beta, \gamma, n}^{2}}\right) \text { and } \mathcal{Q}_{n}=\prod_{n \geq 1}\left(\lambda_{\alpha, \beta, \gamma, n}-x\right)
$$

Since $\lambda_{\alpha, \beta, \gamma, n}$ 's are all positive, it is clear that $\mathcal{P}_{n}>0$ for all $x \in \mathbb{R}^{+} \cup\{0\}$. Also, from the following chain of inequalities

$$
0<\lambda_{\alpha, \beta, \gamma, 1}<\lambda_{\alpha, \beta, \gamma, 2}<\lambda_{\alpha, \beta, \gamma, 3}<\cdots<\lambda_{\alpha, \beta, \gamma, n}<\cdots,
$$

for the zeros, we can make the following observations: if $x \in\left(\lambda_{\alpha, \beta, \gamma, 2 n-1}, \lambda_{\alpha, \beta, \gamma, 2 n}\right)$, then the first $(2 n-1)$ terms of $\mathcal{Q}_{n}$ is strictly negative and the rest is strictly positive. On the other hand, if $x \in\left(\lambda_{\alpha, \beta, \gamma, 2 n}, \lambda_{\alpha, \beta, \gamma, 2 n+1}\right)$, then the first (2n) terms of $\mathcal{Q}_{n}$ is strictly negative and the remained terms are strictly positive. Moreover, it is clear that all the terms of $\mathcal{Q}_{n}$ are strictly positive for $x \in\left[0, \lambda_{\alpha, \beta, \gamma, 1}\right)$. In conclusion, we deduce that the function $x \mapsto \Phi(\alpha, \beta, \gamma, x)$ is negative on $A_{1}$ and it is positive on $A_{3}$.

ii. It is clear from the part $i$. that $\Phi(\alpha, \beta, \gamma, x)>0$ for all $x \in\left[0, \lambda_{\alpha, \beta, \gamma, 1}\right)$. In order to prove the function $x \mapsto \Phi(\alpha, \beta, \gamma, x)$ is a decreasing function on the interval $(0, \lambda, \beta, \gamma, 1)$, it is sufficient 
to show that $\Phi^{\prime}(\alpha, \beta, \gamma, x)<0$ for all $x \in\left(0, \lambda_{\alpha, \beta, \gamma, 1}\right)$. For this purpose, considering the logarithmic derivative of (5). So, using the basic properties of logarithm function we can write that

$$
\frac{\Phi^{\prime}(\alpha, \beta, \gamma, x)}{\Phi(\alpha, \beta, \gamma, x)}=\sum_{n \geq 1} \frac{2 x}{x^{2}-\lambda_{\alpha, \beta, \gamma, n}^{2}}
$$

or

$$
\Phi^{\prime}(\alpha, \beta, \gamma, x)=\Phi(\alpha, \beta, \gamma, x) \sum_{n \geq 1} \frac{2 x}{x^{2}-\lambda_{\alpha, \beta, \gamma, n}^{2}}
$$

It is easy to check that

$$
\sum_{n \geq 1} \frac{2 x}{x^{2}-\lambda_{\alpha, \beta, \gamma, n}^{2}}<0
$$

for all $x \in\left(0, \lambda_{\alpha, \beta, \gamma, 1}\right)$. As a consequence, $\Phi^{\prime}(\alpha, \beta, \gamma, x)<0$ for $x \in\left(0, \lambda_{\alpha, \beta, \gamma, 1}\right)$ which is desired.

iii. In order to prove this assertion, second condition in Definition 1.3 can be used. Namely, we will show that $[\log \Phi(\alpha, \beta, \gamma, x)]^{\prime \prime}<0$ for all $x \in A_{3}$. Therefore, by utilizing the infinite product representation given by (5) we can write that

$$
\begin{aligned}
\frac{d^{2}}{d x^{2}}[\log \Phi(\alpha, \beta, \gamma, x)] & =\frac{d^{2}}{d x^{2}} \log \prod_{n \geq 1}\left(1-\frac{x^{2}}{\lambda_{\alpha, \beta, \gamma, n}^{2}}\right) \\
& =\frac{d^{2}}{d x^{2}} \sum_{n \geq 1} \log \left(1-\frac{x^{2}}{\lambda_{\alpha, \beta, \gamma, n}^{2}}\right) \\
& =\frac{d}{d x} \sum_{n \geq 1} \frac{-2 x}{\lambda_{\alpha, \beta, \gamma, n}^{2}-x^{2}} \\
& =-2 \sum_{n \geq 1} \frac{\lambda_{\alpha, \beta, \gamma, n}^{2}+x^{2}}{\left(\lambda_{\alpha, \beta, \gamma, n}^{2}-x^{2}\right)^{2}}
\end{aligned}
$$

Since

$$
\sum_{n \geq 1} \frac{\lambda_{\alpha, \beta, \gamma, n}^{2}+x^{2}}{\left(\lambda_{\alpha, \beta, \gamma, n}^{2}-x^{2}\right)^{2}}>0
$$

for all $x \in A_{3}$, we can say that $[\log \Phi(\alpha, \beta, \gamma, x)]^{\prime \prime}<0$ for all $x \in A_{3}$ and the function $x \mapsto$ $\Phi(\alpha, \beta, \gamma, x)$ is strictly logarithmic concave on $A_{3}$.

Taking $\gamma=1$ in Theorem 2.1, we get the following results for the two-parameter Mittag-Leffler function: 
Corollary 2.2 Let $\left(\frac{1}{\alpha}, \beta\right) \in W_{i}$ and denote the $n$th positive zero of the two-parameter MittagLeffler function $\phi\left(\alpha, \beta,-x^{2}\right)$ by $\lambda_{\alpha, \beta, n}$. Further, consider the following sets:

$$
B_{1}=\bigcup_{n \geq 1}\left(\lambda_{\alpha, \beta, 2 n-1}, \lambda_{\alpha, \beta, 2 n}\right), B_{2}=\bigcup_{n \geq 1}\left(\lambda_{\alpha, \beta, 2 n}, \lambda_{\alpha, \beta, 2 n+1}\right) \text { and } B_{3}=\left[0, \lambda_{\alpha, \beta, 1}\right) \cup B_{2} .
$$

Then, the two-parameter Mittag-Leffler function $\Phi(\alpha, \beta, x)=\Gamma(\beta) \phi\left(\alpha, \beta,-x^{2}\right)$ has the following properties:

i. $\quad$ the function $x \mapsto \Phi(\alpha, \beta, x)$ is negative on $B_{1}$ and it is positive on $B_{3}$,

ii. the function $x \mapsto \Phi(\alpha, \beta, x)$ is a decreasing function on $\left(0, \lambda_{\alpha, \beta, 1}\right)$,

iii. the function $x \mapsto \Phi(\alpha, \beta, x)$ is strictly logarithmic concave on $B_{3}$.

Taking $\beta=\gamma=1$ in Theorem 2.1, we obtain the following results for the one-parameter Mittag-Leffler function:

Corollary 2.3 Let $\alpha>1$ and denote the $n$th positive zero of the one-parameter Mittag-Leffler function $\phi\left(\alpha,-x^{2}\right)$ by $\lambda_{\alpha, n}$. Further, consider the following sets:

$$
C_{1}=\bigcup_{n \geq 1}\left(\lambda_{\alpha, 2 n-1}, \lambda_{\alpha, 2 n}\right), C_{2}=\bigcup_{n \geq 1}\left(\lambda_{\alpha, 2 n}, \lambda_{\alpha, 2 n+1}\right) \text { and } C_{3}=\left[0, \lambda_{\alpha, 1}\right) \cup C_{2}
$$

Then, the one-parameter Mittag-Leffler function $\Phi(\alpha, x)=\Gamma(\beta) \phi\left(\alpha,-x^{2}\right)$ has the following properties:

i. $\quad$ the function $x \mapsto \Phi(\alpha, x)$ is negative on $C_{1}$ and it is positive on $C_{3}$,

ii. $\quad$ the function $x \mapsto \Phi(\alpha, x)$ is a decreasing function on $\left(0, \lambda_{\alpha, 1}\right)$,

iii. the function $x \mapsto \Phi(\alpha, x)$ is strictly logarithmic concave on $C_{3}$.

Theorem 2.4 Suppose that $\alpha>1, \beta>0$ and $\gamma>0$. Let $\lambda_{\alpha, \beta, \gamma, n}$ denote the $n$th positive zero of the function $x \mapsto \phi\left(\alpha, \beta, \gamma,-x^{2}\right)$.Then, the function $x \mapsto \phi\left(\alpha, \beta, \gamma,-x^{2}\right)$ is strictly logarithmic concave on $A_{3}$.

Proof. It is well-known from the definition of Euler's Gamma function that it is positive defined for $\beta>0$. On the other hand, it can be easily checked that multiplying by a positive constant does not change the logarithmic concavity property of a function. Now, rewrite the function $x \mapsto \phi\left(\alpha, \beta, \gamma,-x^{2}\right)$ as follow:

$$
\phi\left(\alpha, \beta, \gamma,-x^{2}\right)=\frac{1}{\Gamma(\beta)} \Phi(\alpha, \beta, \gamma, x)
$$

Since $\frac{1}{\Gamma(\beta)}>0$ for $\beta>0$ and the function $x \mapsto \Phi(\alpha, \beta, \gamma, x)$ is strictly logarithmic concave on $A_{3}$, we conclude that the function $x \mapsto \phi\left(\alpha, \beta, \gamma,-x^{2}\right)$ is strictly logarithmic concave on $A_{3}$.

Taking $\gamma=1$ and $\beta=\gamma=1$, respectively, in Theorem 2.4, we get the following results:

Corollary 2.5 Suppose that $\alpha>1$ and $\beta>0$. Then, the function $\phi\left(\alpha, \beta,-x^{2}\right)$ is strictly logarithmic concave in $B_{3}$, while the function $x \mapsto \phi\left(\alpha,-x^{2}\right)$ is strictly logarithmic concave in $C_{3}$. 
The following is the our last result and it is concerned with the some monotonic properties of a function defined by the three-parameter Mittag-Leffler function and its derivative.

Theorem 2.6 Let suppose that $\alpha, \beta$ and $\gamma$ are positive real numbers. Then, the function

$$
x \mapsto H(\alpha, \beta, \gamma, x)=\frac{x \phi^{\prime}(\alpha, \beta, \gamma, x)}{\phi(\alpha, \beta, \gamma, x)}
$$

is an increasing function on $\mathbb{R}^{+}$.

Proof. By using the infinite sum representation of the function $x \mapsto \phi(\alpha, \beta, \gamma, x)$ given by (3), we may write

$$
H(\alpha, \beta, \gamma, x)=\frac{\sum_{n \geq 0} \delta_{n} x^{n}}{\sum_{n \geq 0} \mathcal{T}_{n} x^{n}}
$$

where $\mathcal{S}_{n}=\frac{n(\gamma)_{n}}{n ! \Gamma(\alpha n+\beta)}$ and $\mathcal{T}_{n}=\frac{(\gamma)_{n}}{n ! \Gamma(\alpha n+\beta)}$. With the help of the Lemma 1.2 it can be shown that the function $x \mapsto H(\alpha, \beta, \gamma, x)$ is an increasing function on $\mathbb{R}^{+}$. It is clear that $\delta_{n} \in \mathbb{R}$ and $\mathcal{T}_{n}>$ 0 for $\alpha>0, \beta>0, \gamma>0$ and $n \in\{0,1,2, \ldots\}$. On the other hand, according to famous CauchyHadamard Theorem, both power series $\sum_{n \geq 0} \delta_{n} x^{n}$ and $\sum_{n \geq 0} \mathcal{T}_{n} x^{n}$ are convergent on $\mathbb{R}$. Now, we want to show that the radius of convergence of the both power series is $\infty$. Let $R_{1}$ and $R_{2}$ denote the radii of convergence of the power series $\sum_{n \geq 0} \mathcal{S}_{n} x^{n}$ and $\sum_{n \geq 0} \mathcal{T}_{n} x^{n}$, respectively. Some basic computations yield that

$$
\begin{aligned}
R_{1} & =\lim _{n \rightarrow \infty}\left|\frac{\mathcal{S}_{n}}{\mathcal{S}_{n+1}}\right|=\lim _{n \rightarrow \infty}\left|\frac{\frac{n(\gamma)_{n}}{n ! \Gamma(\alpha n+\beta)}}{\frac{(n+1)(\gamma)_{n+1}}{(n+1) ! \Gamma(\alpha(n+1)+\beta)}}\right| \\
& =\lim _{n \rightarrow \infty} \frac{n \frac{\Gamma(\gamma+n)}{\Gamma(\gamma)} \Gamma(\alpha n+\alpha+\beta)}{\Gamma(\alpha n+\beta) \frac{\Gamma(\gamma+n+1)}{\Gamma(\gamma)}} \\
& =\lim _{n \rightarrow \infty} \frac{\Gamma(\alpha n+\alpha+\beta)}{\Gamma(\alpha n+\beta)} .
\end{aligned}
$$

The following asymptotic relation known as Stirling formulae

$$
\Gamma(x+1) \sim \sqrt{2 \pi x} x^{x} e^{-x}, \quad x \rightarrow \infty
$$

can be used to compute the limit in (6). Taking $x=\alpha n+\beta-1$ in (7) and using the asymptotic relation $\left(1+\frac{a}{x}\right)^{x} \sim e^{a}$ for $x \rightarrow \infty$, we deduce that

$$
\Gamma(\alpha n+\beta) \sim \sqrt{2 \pi}(\alpha n+\beta)^{\alpha n+\beta-\frac{1}{2}} e^{-(\alpha n+\beta)}, \quad n \rightarrow \infty .
$$

Now, considering the relation (8) in (6) one can easily obtain

$$
R_{1}=\lim _{n \rightarrow \infty} \frac{\Gamma(\alpha n+\alpha+\beta)}{\Gamma(\alpha n+\beta)}=\lim _{n \rightarrow \infty} \frac{\sqrt{2 \pi}(\alpha n+\beta+\alpha)^{\alpha n+\beta+\alpha-\frac{1}{2}} e^{-(\alpha n+\beta+\alpha)}}{\sqrt{2 \pi}(\alpha n+\beta)^{\alpha n+\beta-\frac{1}{2}} e^{-(\alpha n+\beta)}}
$$




$$
=e^{-\alpha} \lim _{n \rightarrow \infty}\left(1+\frac{\alpha}{\alpha n+\beta}\right)^{\alpha n+\beta+\alpha-\frac{1}{2}} \lim _{n \rightarrow \infty}(\alpha n+\beta)^{\alpha}=\infty .
$$

By following the similar procedure one can easily show that $R_{2}=\infty$. As a result, we can say that both power series $\sum_{n \geq 0} \mathcal{S}_{n} x^{n}$ and $\sum_{n \geq 0} \mathcal{T}_{n} x^{n}$ are convergent on $\mathbb{R}$. Now, if we consider the sequence $\mathcal{U}_{n}=\frac{\delta_{n}}{\mathcal{J}_{n}}=n$, then we can say that the sequence $\left\{\mathcal{U}_{n}\right\}_{n \geq 0}$ is an increasing sequence since $\frac{u_{n+1}}{u_{n}}=\frac{n+1}{n}>1$. By applying Lemma 1.2 we complete the proof.

\section{Applications}

This section is devoted for some applications of our results. It is known that, just like other special functions, three parameter Mittag-Leffler function reduces to the basic functions like exponential, trigonometric, hyperbolic and so on. Especially, using Taylor-Maclaurin series expansions of basic functions, many special cases of the functions $\phi, \Phi$ and $H$ can be obtained for the special values of $\alpha, \beta$ and $\gamma$. Some of these functions are given below:

- $\phi\left(2,1,1,-x^{2}\right)=\Phi(2,1,1, x)=\cos x, \quad H(2,1,1, x)=\frac{1}{2} \sqrt{x} \tanh \sqrt{x}$.

- $\phi\left(2,3,1,-x^{2}\right)=\frac{1-\cos x}{x^{2}}, \quad \Phi(2,3,1, x)=\frac{2(1-\cos x)}{x^{2}}, \quad H(2,3,1, x)=\frac{\sqrt{x} \sinh \sqrt{x}-2 \cosh \sqrt{x}+2}{2(\cosh \sqrt{x}-1)}$.

- $\phi\left(2,1,2,-x^{2}\right)=\Phi(2,1,2, x)=\cos x-\frac{x \sin x}{2}, \quad H(2,1,2, x)=\frac{3 \sqrt{x} \sinh \sqrt{x}+x \cosh \sqrt{x}}{2 \sqrt{x} \sinh \sqrt{x}+4 \cosh \sqrt{x}}$.

- $\phi\left(2,4,1,-x^{2}\right)=\frac{x-\sin x}{x^{3}}, \quad \Phi(2,4,1, x)=\frac{6(x-\sin x)}{x^{3}}, \quad H(2,4,1, x)=\frac{x \cosh \sqrt{x}-3 \sqrt{x} \sinh \sqrt{x}+2 x}{2 \sqrt{x}(\sinh \sqrt{x}-\sqrt{x})}$.

It is possible to determine the sign, monotonicity and logarithmic concavity properties of the above mentioned functions by taking some special values for the parameters in Theorem 2.1, Theorem 2.4 and Theorem 2.6. Some of these examples are given below:

Example 3.1 The following statements hold true.

a. The function $x \mapsto \Phi(2,1,1, x)=\cos x$ is negative on $\kappa_{1}=\bigcup_{n \geq 1}\left(\lambda_{2,1,1,2 n-1}, \lambda_{2,1,1,2 n}\right)$ and it is positive on $\kappa_{3}=\left[0, \lambda_{2,1,1,1}\right) \cup \kappa_{2}$, where $\kappa_{2}=U_{n \geq 1}\left(\lambda_{2,1,1,2 n}, \lambda_{2,1,1,2 n+1}\right)$ and $\lambda_{2,1,1, n}$ shows the $n$th positive root of the equation $\cos x=0$.

b. The function $x \mapsto \Phi(2,1,2, x)=\cos x-\frac{x \sin x}{2}$ is negative on $\xi_{1}=$ $\cup_{n \geq 1}\left(\lambda_{2,1,2,2 n-1}, \lambda_{2,1,2,2 n}\right)$ and it is positive on $\xi_{3}=\left[0, \lambda_{2,1,2,1}\right) \cup \xi_{2}$, where $\xi_{2}=$ $\mathrm{U}_{n \geq 1}\left(\lambda_{2,1,2,2 n}, \lambda_{2,1,2,2 n+1}\right)$ and $\lambda_{2,1,2, n}$ shows the $n$th positive root of the equation $2 \cos x-x \sin x=0$.

Example 3.2 The following assertions are valid.

a. The function $x \mapsto \Phi(2,1,1, x)=\cos x$ is a decreasing function on $\left(0, \lambda_{2,1,1,1}\right)$.

b. The function $x \mapsto \Phi(2,3,1, x)=\frac{2(1-\cos x)}{x^{2}}$ is a decreasing function on $\left(0, \lambda_{2,3,1,1}\right)$.

c. The function $x \mapsto \Phi(2,1,2, x)=\cos x-\frac{x \sin x}{2}$ is a decreasing function on $\left(0, \lambda_{2,1,2,1}\right)$.

Example 3.3 The following statements hold true.

a. The function $x \mapsto \Phi(2,1,1, x)=\cos x$ is strictly logarithmic concave on the set $\left[0, \lambda_{2,1,1,1}\right) \cup \kappa_{1}$, where $\kappa_{1}=\bigcup_{n \geq 1}\left(\lambda_{2,1,1,2 n}, \lambda_{2,1,1,2 n+1}\right)$ and $\lambda_{2,1,1, n}$ shows the $n$th positive root of the equation $\cos x=0$. 
b. The function $x \mapsto \Phi(2,3,1, x)=\frac{2(1-\cos x)}{x^{2}}$ is strictly logarithmic concave on the set $\left[0, \lambda_{2,3,1,1}\right) \cup \kappa_{2}$, where $\kappa_{2}=\bigcup_{n \geq 1}\left(\lambda_{2,3,1,2 n}, \lambda_{2,3,1,2 n+1}\right)$ and $\lambda_{2,3,1, n}$ shows the $n$th positive root of the equation $\cos x=1$.

c. The function $x \mapsto \Phi(2,1,2, x)=\cos x-\frac{x \sin x}{2}$ is strictly logarithmic concave on the set $\left[0, \lambda_{2,1,2,1}\right) \cup \kappa_{3}$, where $\kappa_{3}=\bigcup_{n \geq 1}\left(\lambda_{2,1,2,2 n}, \lambda_{2,1,2,2 n+1}\right)$ and $\lambda_{2,1,2, n}$ shows the $n$th positive root of the equation $2 \cos x-x \sin x=0$.

d. The function $x \mapsto \Phi(2,4,1, x)=\frac{6(x-\sin x)}{x^{3}}$ is strictly logarithmic concave on the set $\left[0, \lambda_{2,4,1,1}\right) \cup \kappa_{4}$, where $\kappa_{4}=\bigcup_{n \geq 1}\left(\lambda_{2,4,1,2 n}, \lambda_{2,4,1,2 n+1}\right)$ and $\lambda_{2,4,1, n}$ shows the $n$th positive root of the equation $\sin x=x$.

Example 3.4 Each of the following functions

$$
\begin{gathered}
H(2,1,1, x)=\frac{1}{2} \sqrt{x} \tanh \sqrt{x}, \quad H(2,3,1, x)=\frac{\sqrt{x} \sinh \sqrt{x}-2 \cosh \sqrt{x}+2}{2(\cosh \sqrt{x}-1)}, \\
H(2,1,2, x)=\frac{3 \sqrt{x} \sinh \sqrt{x}+x \cosh \sqrt{x}}{2 \sqrt{x} \sinh \sqrt{x}+4 \cosh \sqrt{x}} \text { and } H(2,4,1, x)=\frac{x \cosh \sqrt{x}-3 \sqrt{x} \sinh \sqrt{x}+2 x}{2 \sqrt{x}(\sinh \sqrt{x}-\sqrt{x})}
\end{gathered}
$$

is an increasing function on $\mathbb{R}^{+}$.

\section{References}

[1] Baricz, Á., Prajapati, A., "Radii of starlikeness and convexity of generalized MittagLeffler functions”, Mathematical Communication 25(1) (2020) : 117-135.

[2] Biernacki, M., Krzyż, J., "On the monotonity of certain functionals in the theory of analytic functions”, Annales Universitatis Mariae Curie-Sklodowska, sectio A 9 (1955) : 135-147.

[3] Gorenflo, R., Kilbas, A. A., Mainardi, F., Rogosin, S. V., "Mittag-Leffler functions, related topics and applications”, Springer, 2014.

[4] Gupta, I. S., Debnath, L., "Some properties of the Mittag-Leffler functions”, Integral Transforms and Special Functions 18(5) (2007) : 329-336.

[5] Kumar, H., Pathan, M. A., "On the distribution of non-zero zeros of generalized MittagLeffler functions”, International Journal of Engineering Research and Application 6(10) (2016) : 66-71.

[6] Borzadaran, G. M., Borzadaran, H. M., "Log-concavity property for some well-known distributions”, Surveys in Mathematics and its Applications 6 (2011) : 203-19.

[7] Peresyolkova, I. N., "On distribution of zeros of generalized functions of Mittag-Leffler type”, Matematychni Studii 13(2) (2000) : 157-64.

[8] Prabhakar, T. R., “A singular integral equation with a generalized Mittag Leffler function in the kernel”, Yokohama Mathematical Journal 19 (1971) : 7-15.

[9] Wiman, A., "Über die Nullstellen der Funktionen $E_{a}(x)$ ", Acta Mathematica 29(1) (1905) : 217-34. 